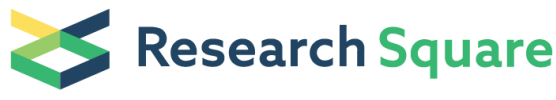 \\ Preprints are preliminary reports that have not undergone peer review. \\ They should not be considered conclusive, used to inform clinical practice, or referenced by the media as validated information.
}

\section{Monogenic autoimmunity caused by TLR7 gain-of-function}

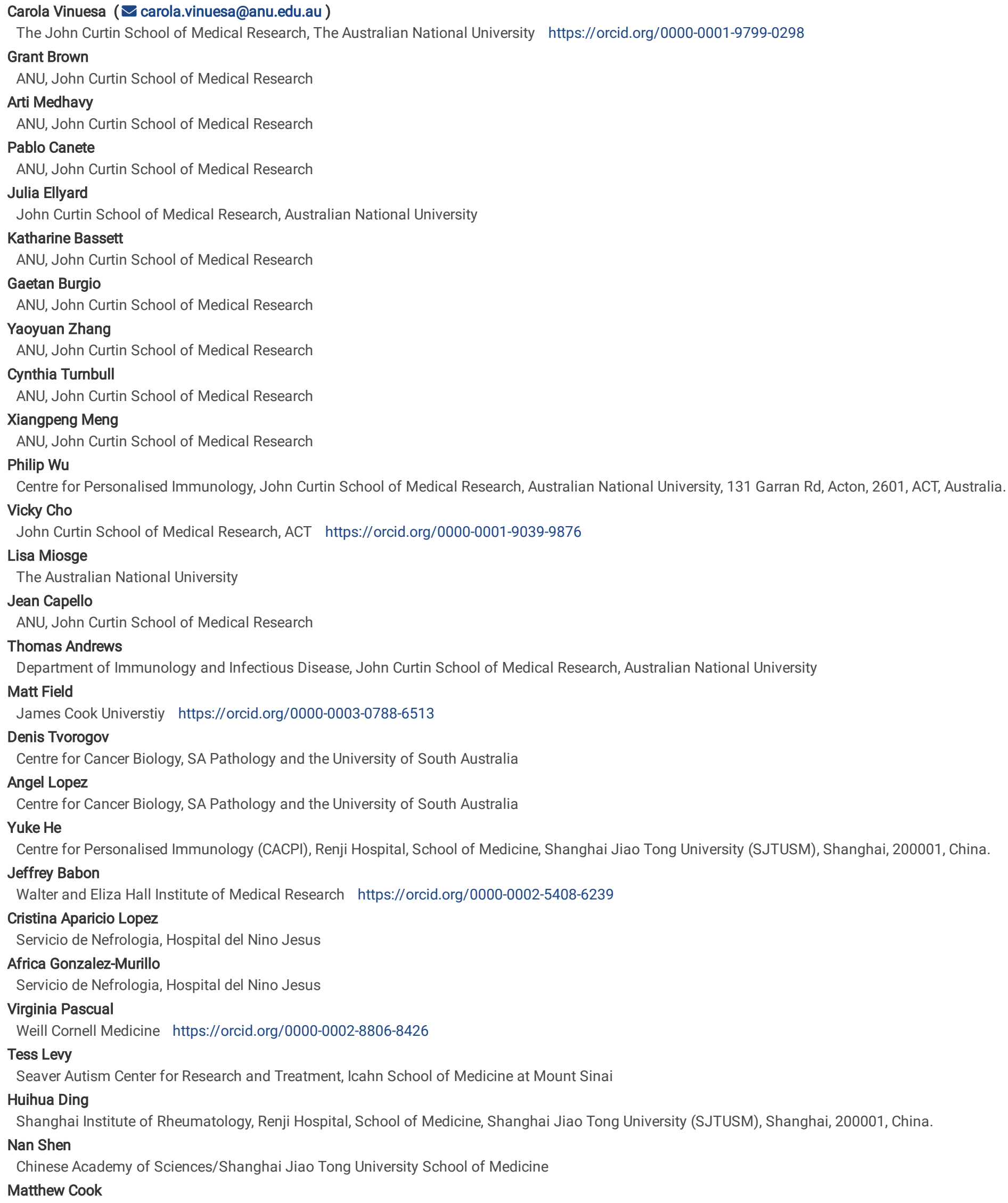


ANU, John Curtin School of Medical Research

Eric Mallack

Division of Child Neurology, Weill Cornell Medical College, NewYork-Presbyterian Hospital

Carmen de Lucas Collantes

Servicio de Nefrologia, Hospital del Nino Jesus

Vicky Athanasopoulos

China Australia Centre for Personalised Immunology, Australian National University and Shanghai Renji Hospital, JiaoTong University of Shanghai.

\section{Biological Sciences - Article}

Keywords: TLR7 signaling, pathogenesis, human systemic autoimmune disease

Posted Date: January 27th, 2021

DOI: https://doi.org/10.21203/rs.3.rs-152145/v1

License: @ (i) This work is licensed under a Creative Commons Attribution 4.0 International License. Read Full License 


\section{Abstract}

While circumstantial evidence supports enhanced TLR7 signaling as a mechanism of human systemic autoimmune disease, we have lacked the proof afforded by lupus-causing TLR7 gene variants. Here we describe monogenic human systemic lupus erythematosus (SLE) caused by TLR7 gain-of-function. We identified a de novo, novel, missense TLR7Y264H variant in a child with severe lupus and additional novel or rare variants in probands with interferonopathies or systemic autoimmunity (Aicardi Goutieres Sd, SLE, Sjogren's Sd, and juvenile idiopathic arthritis). The variants increased NF-KB and IFN$\beta$ activity and the de novo TLR7Y264H variant was sufficient to cause lupus when introduced in mice. We show that constitutive TLR7 signaling drives aberrant survival of BCR-activated B cells that would otherwise die, and accumulation of CD11 $\mathrm{C}^{+}$age-associated B cells and germinal center (GC) B cells in a B cell-intrinsic manner. Follicular and extrafollicular helper T-cells were also increased but these phenotypes were cell-extrinsic. MyD88-deficiency rescued autoimmunity, aberrant B cell survival, and all cellular and serological phenotypes. Despite prominent spontaneous GC formation in mice carrying the TLR7 Y264H variant, we show that TLR7-driven lupus was not ameliorated when the TLR7 Y264H mice were made GC-deficient suggesting extrafollicular origin of pathogenic B cells. We establish the importance of TLR7 for human SLE pathogenesis, which paves the way for therapeutic TLR7 or MyD88 inhibition.

\section{Main Text}

SLE is generally considered to be a polygenic autoimmune disease, although in some cases, rare alleles have been identified that have provided important insights into disease mechanisms $(1,2)$. The discovery of monogenic lupus cases has highlighted the importance of complement, type I interferons, TLR7 ligation, and B cell survival in disease pathogenesis (3). There is accumulating evidence that human SLE patients display phenotypes consistent with increased TLR7 signaling, with elevated IgD- $\mathrm{CD}^{-} 7^{-}$(double negative, DN) B cells, and more specifically, the CXCR5 ${ }^{-}$CD11 ${ }^{+}$subset (also known as DN2 B cells or age-associated B cells (ABCs)) in peripheral blood (4) and excessive accumulation of extra-follicular helper T cells (5). Genome wide association studies (GWAS) have identified common polymorphisms in or near TLR7 that segregate with SLE (6-8). In mice, increased TLR7 signaling due to the Yaa duplication or to transgenic $T L R 7$ expression has been shown to exacerbate autoimmunity to RNA-related self-antigens typical of lupus and Sjogren's syndrome ( 9,10$)$. In the same line, deletion of $T L R 7$ prevents or ameliorates disease in other lupus models, such as the 564lgi transgenic mice in which $B$ cells encode a receptor that binds to RNA ligands (11). Despite this mounting link between TLR7 and the pathogenesis of lupus, no monogenic lupus cases due to TLR7 variants have been reported to date. There is also conflicting evidence as to how TLR7 overexpression causes autoimmunity, particularly, the relative roles of TLR7-driven spontaneous germinal centers versus the role of TLR7-driven DN B cells; the latter have been proposed to originate extrafollicularly and be pathogenic in lupus (4). Most mouse lupus models in which TLR7 plays a role in pathogenicity display increased GC and Tfh formation $(9,10)$ and it has been proposed that TLR7 drives germinal centers enriched in self-reactive B cells (12). However, recent reports have demonstrated that lupus can develop independently of GCs in mouse models in which disease is dependent on MyD88 signaling $(13,14)$.

TLR7 has been considered to be predominantly a receptor for single stranded RNA and it typically gets activated by guanosine-and uridine-rich RNA, with guanosine binding to site 1 and polyuridine to site 2 (15). RNA viruses such as HIV, influenza, vesicular stomatitis and more recently SARS-CoV2 have been suggested to activate TLR7 (16-19). Reports over the last few years have suggested that the endogenous ligand for TLR7 is smRNP (20,21) which contains the TLR7 stimulatory U1 RNA molecule (22). Solving TLR7's crystal structure revealed that TLR7 binds to endogenously-produced 2'3'GMP, a cyclic nucleotide derived from GTP degradation (15), at the site (site 1) recognized by the ligands commonly used to activate TLR7 (R837, R848). Binding of R848 to site 1 is enough to trigger signaling, without the need for poly-Uridine binding to site 2 (15). Here, we describe the action of a novel TLR7gain-of-function (GoF) variant that occurs in a residue that binds 2'3'-GMP, causing constitutive TLR7 activation and monogenic childhood-onset SLE. We report additional novel and ultrarare GoF variants in another five patients and show how this pathway contributes to autoimmunity.

\section{Novel and ultrarare TLR7 variants in autoimmune patients}

We undertook whole genome sequencing (WES) of a Spanish girl who had presented at age 7 with refractory immune thrombocytopenia and hemichorea. She was found to have renal involvement after admission with a hypertensive crisis (140-150 / 80 mmHg > P99 for her height and sex) that required treatment with enalapril, amlodipine and spironolactone. She was also found to have mild mitral insufficiency and severe thrombocytopenia (lowest of $8.000 / \mu \mathrm{L}$ ). She also suffered intermittent episodes of chorea. Bioinformatic analysis revealed a de novo, TLR7p.Tyr264His (Y264H) novel missense variant (Fig. 1a-b, Family A and Table S1), predicted to be damaging by SIFT and CADD (Table S2). This variant was not present in the databases of normal human sequence variation (gnomAD, ExAC, dbSNP). Examination of the BAM files (Fig. 1c) together with paternity analysis (Fig. S1) confirmed the mutation occurred de novo. The mutated tyrosine residue lies in the eighth leucine-rich repeat of TLR7 (23), within the endosomal part of the receptor (Fig. 1d) and is highly conserved across species including zebrafish (Fig. 1e).

WES of additional cases of SLE or related autoimmune diseases and interferonopathies including Sjogren's Sd, Aicardi-Goutières Sd (AGS) and juvenile idiopathic arthritis (JIA) identified other novel (C.II.1 R28G), ultrarare (B.II.1 E906K; D.I.1 S724R) and rare (E.II.1, A448V) variants in TLR7 (Fig. 1a, d, e and Tables S1, S2). Of these, the most severe case was that of B.II.1, a North American girl diagnosed clinically with interferon-related encephalitis/AGS spectrum disease at 2.5 years of age. Within 6 months she developed progressive ascending hypertonicity, hyperreflexia, and weakness leading to gross motor regression (loss of ability to walk or sit independently) and expressive language regression to a non-verbal state. Fine motor skills were less affected, with some fisting and cortical thumbing. Her neurological disease was preceded 1-2 months prior by a month-long febrile illness. Lumbar puncture excluded viral encephalitis and she was found to have an interferon signature with an ISG expression score that was 2.5 to 6.6 -fold above the controls ( $p<0.05$ ) (data not shown). Sequencing identified a maternally inherited heterozygous TLR7 missense p.Glu906Lys (E906K) variant, predicted to be damaging by PolyPhen-2 and SIFT. She also carried a paternally-inherited novel variant in MYD88: c.16_34del, p.Ala6Profs*39 (deletion of 19 nucleotides from positions 16 to 34 , predicted to result in an A6P substitution and a new reading frame with a stop codon 39 amino acids downstream).

\section{TLR7variants are gain-of-function}


Flow cytometric analysis of PBMCs from the probands with the de novo and ultrarare $T L R 7$ variants revealed elevated plasmablasts with variable proportions of memory B cells (Fig. 1f) and elevated CD19hi IgD` CD27 CD21 lo B cells also known as double negative (DN) B cells (Fig. 1g); the latter have been associated with increased TLR7 signaling (4). The most significant increases in plasmablasts and DN B cells were noted in children A.II.1 with SLE and the TLR7Y264H de novo variant and B.II.1 with AGS carrying the combination of TLR7E906K and MYD88 A6Pfs39* variants.

We speculated pathogenic variants would enhance TLR7 signaling and therefore downstream NF-kB and IFN-b activation. To test this, we transfected RAW 264.7 macrophage-like cells with plasmids encoding the different TLR7 mutants together with MYD88. Compared with wild type, overexpression of TLR7 constructs showed enhanced NF-kB and IFN-b activity for most mutant variants suggesting priming of TLR7 for enhanced activation. Interestingly, the de novo Y264H variant did not show increased activity (Fig. 1h), suggesting that it was constitutively active and either refractory to exogenous or over-expressioninduced stimulation or was toxic $(24,25)$ (see below). Activation of transfected cells with the TLR7 agonists R837 and R848 showed similar results (Fig. S1c). We also expressed the truncated A6Pfs39* MYD88 found in proband B.II.1 and found it enhanced IFN-b signaling while it dampened NF-kB (Fig. 1i).

\section{TLR7 Y264 causes autoimmunity in mice}

Given the different luciferase assay results with the $\mathrm{Y} 264 \mathrm{H}$ variant, we were intrigued by the $T L R 7 \mathrm{Y} 264 \mathrm{H}$ allele. The proband carrying this allele had a very severe and early onset SLE and the variant occurred de novo, suggesting a possible monogenic form of disease. Using the published TLR7 structure, we mapped the mutated residue to site 1 of TLR7 ligand binding site, where 2'3'-cGMP binds (15) (Fig. 1j). Indeed, the Y264 side-chain OH forms a hydrogen bond with 2'3'-cGMP (15). Upon binding to site 1, small molecules like R837 induce TLR7 dimerization and formation of the active signaling form (26) (Fig 1k). This suggests TLR7 Y264H substitution gives rise to a constitutive, maximally activated form, likely to be toxic to RAW264.7 cells: high albeit therapeutic doses of TLR7 agonists drive apoptosis of cancer cells $(24,25)$.

To investigate whether the TLR7Y264H allele could cause monogenic SLE we introduced the orthologous allele into mice using CRISPR/Cas9 editing. WES of affected and unaffected male littermates confirmed the T/r7Y264H was the only relevant CRISPR induced coding variant that segregated with the phenotype. The resulting strain was named "kika" by the girl carrying the TLR7Y264H variant (A.II.1), with TIr7Y264H being abbreviated as the "kik" allele henceforth. A strain we generated lacking TLR7 protein due to a $1 \mathrm{bp}$ deletion was included as a control (Fig. S2a-b). Twelve-week-old mice carrying one or both kika alleles displayed splenomegaly (Fig. 2a). Kika mice also had decreased survival (Fig. 2c).

We next evaluated the presence of autoantibodies. Male T/r $7^{k i k / y}$ and female heterozygous $T / r{ }^{k i k /+} k i k a$ mice developed autoantibodies to ssDNA, ssRNA and SmRNP; the latter two being reported to be TLR7 ligands (17) (Fig. 2d). All mice carrying kika alleles were ANA-positive by HEp-2 immunofluorescence (Fig. 2e) with nuclear, cytoplasmic, cell-cycle-dependent and Golgi staining (Fig. 2f). Weak dsDNA reactivity was present in 10-20\% of kika mice as assessed by Crithidia luciliae immunofluorescence (Fig. S3).

As seen in proband A.II.1, thrombocytopenia was marked in heterozygous TIr $7^{k i k /+}$ kika mice (Fig. 2g), which also had slightly lower white blood cell count (Fig. S4a). Proliferative glomerulonephritis was evident in kidneys (Fig. 2h), with accumulation of electron-dense immunoglobulin deposits as assessed by electron microscopy (Fig. 2i). Lymphoid infiltrates were seen in the liver (Fig. 2j, k) and pancreas (Fig. 2l, m), which contained large peri-islet infiltrates occasionally forming follicular structures (Fig. 2m). Exocrine pancreatic tissue was often replaced by fat, particularly in homozygous T/r $7^{k i k / k i k} \mathrm{mice}$ (Fig. $2 \mathrm{n}$ ).

\section{TLR7 Y264H causes cell-intrinsic expansion of effector B cells}

Flow cytometric analysis of kika spleens revealed a reduced T:B ratio (Fig. S4b): total B cells were increased by a median of 1.7-fold in both heterozygous and homozygous female kika mice, whereas total T cell numbers did not change (data not shown). Analysis of B cell subsets revealed marked spontaneous GC formation in spleens (Fig 3a) as well as increased ABCs in both spleen (Fig. 3b) and blood (Fig. S4c). Splenic marginal zone B cells were decreased in percentages but not in total numbers (Fig. S4d). Both Tfh cells and CXCR3 ${ }^{+}$extrafollicular helper CD4 ${ }^{+} \mathrm{T}$ (eTh) were elevated in kika mice (Fig. 1c, d). Strikingly, kika mice had circulating Tfh cells in the blood expressing high amounts of PD-1 and CXCR5, comparable to splenic GC-Tfh cells (Fig. S4e). Plasma cells (Fig. 3e) and effector/memory CD $4^{+} \mathrm{CD} 44^{\text {hi }} \mathrm{T}$ cells (Fig 3f) were also expanded in kika spleens. Plasmacytoid dendritic cells (pDCs) appeared to be activated with increased MHCII and reduced Siglec-H expression (27) (Fig S4f). By contrast, TLR7-deficient mice had opposite phenotypes, lacking spontaneous GC B cell, Tfh cells and ABCs, and showing reduced plasma cells (Fig. S4g). In general, the cellular phenotypes of female mice carrying either one or two kika alleles were largely comparable.

We then set out to establish which cellular phenotypes were cell-autonomous. Mixed bone marrow chimeras were generated by adoptively transferring $50: 50$ mixes of either WT CD 45.1: kika CD45.2 or WT CD45.1:WT CD45.2 bone marrow into sub-lethally irradiated Rag $1 \%$ mice. Examination of spleens 22 weeks post-reconstitution revealed that TLR7 acts B cell-intrinsically to promote increased formation of GCs, ABCs and plasma cells (Fig. 3g). By contrast, accumulation of effector $\mathrm{CD} 4^{+} \mathrm{T}$ cells, Tregs, Tfh cells and eTh and reduction in MZ B cells were largely extrinsic, with comparable changes in mutant and wild type cells (Fig. $3 g$ and Fig. S4h).

Proband A.II.1 was also heterozygous for another rare variant in a gene associated with SLE: RNASEH2B, p.Ala177Thr, which, when homozygous, causes SLE and AGS (28). RNASHE2B activates CGAS-STING which can increase type 1 IFN production and TLR7 signaling $(29,30)$. We therefore wondered whether a single Rnaseh $2 b$ loss-of-function allele might exacerbate the phenotype conferred by $T L R 7 Y 264 \mathrm{H}$. To test this, we generated mice hemizygous for a Rnaseh $2 b 1$ base pair deletion leading to a premature stop codon at amino acid 175 (Fig. S5a). Heterozygous mice were viable and had no immunological phenotypes whereas as previously reported, mice homozygous for Rnaseh2b deletion were embryonically lethal (31) (Fig. S5b). Evaluation of $R$ naseh $2 b^{+/-}$ $T / r 7^{k i k /+}$ double heterozygous female mice revealed that Rnaseh2b hemizygosity did not exacerbate the phenotype of $T / r+k i k /++$ female mice (Fig. S5c) suggesting that TLR7 GoF alone can cause monogenic SLE. 


\section{TLR7 GoF allele promotes survival of BCR-activated B cells}

Next, we investigated the mechanism by which the kik allele alters TLR7 function. First, we asked whether the TLR7 Y264H variant induces constitutive TLR7 activation, which can be assessed by the presence of spontaneous TLR7 cleavage in the absence of stimulation (26). Western blots of splenocytes from TLR7 from kika and wild-type littermates using two different antibodies against both $\mathrm{C}$ and $\mathrm{N}$ termini revealed the presence of the $\sim 75 \mathrm{kda} \mathrm{kDa} C$ terminal and $\sim 60 \mathrm{kda} \mathrm{kDa} \mathrm{N}$ terminal cleaved TLR7 product in splenocytes from unimmunized kika mice (Fig. 4a). Such cleaved fragment has been reported to be the active form of TLR7 (24).

We then explored the stage at which TLR7 Y264H breaks B cell tolerance. We hypothesized that constitutive TLR7 signaling may provide an aberrant signal 2 to self-reactive B cells that have bound self-antigen through their BCR (signal 1) and would otherwise die within 72 hours, as occurs in anergic B cells (31) and in immature $\mathrm{CD} 3^{+} \mathrm{B}$ cells stimulated with a-IgM (32). We could not separately activate immature/transitional $\mathrm{B}$ cells from spleen, because we found that agonistic TLR7 treatment of mature splenic B cells upregulates CD93 (Fig. S6a). Not surprisingly, CD93 ${ }^{+}$B cells were abundant in kika mice and it was not possible to determine what fraction of these were truly immature. Hence, we activated total MACS-purified splenic B cells from kika, WT littermates and Yaa mice carrying a $T L R 7$ duplication $(10,11)$, stimulated them with R837 or a-IgM and performed live cell counts 72 hours later. We observed that a-lgM, but not R837, enhanced survival of both total and mature kika B cells compared to control cells (Fig. 4b and S6b). This differs from reports using TLR7 transgenic B cells, which only displayed increased survival when activated with a TLR ligand and not with a-lgM (32), again suggesting constitutive activation of TLR7 $\mathrm{Y} 264 \mathrm{H}$. Given that B cell development and proportions of immature B cells in the bone marrow were largely comparable between kika and control littermates (Fig. S6c) we looked at a-lgM-induced survival in this compartment. Increased survival was also observed in sorted bone marrow immature kika B cells (CD93 ${ }^{+}$ B220 int) when stimulated with a-lgM (Fig. 4c). These results suggest that constitutive TLR7 signaling allows the survival of B cells that are binding selfantigen through their surface BCR.

\section{Autoimmunity in TLR7 Y264H mice is MyD88-dependent and GC-independent}

To confirm that the observed aberrant B cell survival upon IgM stimulation was due to constitutive TLR7 signaling, we crossed kika mice to Myd88 knockout mice to generate $k i k a . M y d 88^{-/}$mice. MyD88 deficiency completely rescued each and every kika phenotype including splenomegaly (Fig. 4d), accumulation of $\mathrm{ABC}\left(\mathrm{CD} 23^{\mathrm{lo}}, \mathrm{CXCR}^{\mathrm{lo}}, \mathrm{CD} 11 \mathrm{c}^{+}, \mathrm{CD} 19^{\mathrm{hi}}\right), \mathrm{GC}$ B, PC, eTh (Fig. 4e-i and Fig. S7) and autoantibody formation (Fig. 4j) confirming GoF TLR7 signaling via MyD88 causes the autoimmune phenotype. Consistent with constitutive TLR7 signaling being responsible for the aberrant survival of $B$ cells receiving only signal 1 (BCR), the enhanced survival was completely abrogated in a-lgM-activated kika B cells lacking MyD88 (Fig. 4k). Concomitant cell-trace violet dye dilution analysis excluded increased proliferation as the cause of increased B cell numbers in the kika cultures (Fig. 4l).

It remains controversial whether the spontaneous GCs of lupus-prone mice contribute to the autoimmune phenotype. This is particularly the case for TLR7driven autoimmunity, where GCs are abundant. There are opposing views with some suggesting that TLR7 promotes the appearance of self-reactive GC B cells that give rise to the autoantibodies (12) and others proposing that the pathogenic $B$ cells are $A B C$ cells of extrafollicular origin (4). To resolve this question, we crossed kika mice to $\mathrm{Bcl} 6^{\text {flox/flox }}$. $\mathrm{CD} 23^{\mathrm{Cre}}$ mice that cannot form germinal centers.

In the F2-intercross offspring, we enumerated GC B cells and confirmed that kika Bcl6flox/flox. CD $23^{\text {Cre }}$ mice had a profound reduction in GC B cells (Fig. $4 \mathrm{~m}$ ). Despite the paucity of GC B cells, kika Bcl $6^{\text {flox/flox }} \cdot \mathrm{CD} 23^{\mathrm{Cre}}$ mice developed the autoimmune phenotype observed in GC-forming littermate kika mice. This included expansion of ABCs, PC (Fig. 4n), eTh and Tfh (Fig. 4o) and formation of autoantibodies to DNA, RNA and SmRNP (Fig. 4p). These results indicate that TLR7-driven autoimmunity can be GC-independent.

We conclude that TLR7 GoF can cause monogenic SLE as well as contribute to polygenic human autoimmunity. TLR7 Y264 directly interacts with the recently described endogenous ligand 2'3'-cGMP (15). The Y264H substitution may thus create a structural change at site 1 or increase the binding affinity for 2' 3 'CGMP, leading to constitutive TLR7 activation -R848 binding at this site (site 1) has been shown to be enough to trigger signaling without the need of polyUridine binding to site 2. Analysis of the mouse model revealed that TLR7 plays a crucial role in promoting the survival of BCR-activated immature B cells. This is likely to be an important tolerance checkpoint given the immature B cells are enriched in self-reactivity (33). Activation of self-reactive B cells by selfantigen in the presence of a constitutive signal 2 is likely to break B cell anergy and lead to differentiation and autoantibody production of B cells that would otherwise be destined to die in the absence of T cell help.

Intriguingly, despite our demonstration that enhanced TLR7 signaling causes cell-autonomous accumulation of both ABCs and GC B cells, we show that GC B cells are dispensable for the autoimmune phenotype. It is therefore likely that extrafollicular $A B C$ s are the main source of pathogenic $B$ cells. It will be important to determine if this is true for all forms of human SLE, or it is restricted to patients in which excessive TLR7 signaling is the dominant pathogenic pathway. Although highly damaging TLR7 GoF mutations are rare, our data, together with evidence of increased TLR7 signaling in a large fraction of SLE patients (4), suggests that TLR7 is a key upstream driver of human SLE. Combinations of gene variants downstream of this receptor causing increased MyD88 signaling are likely to be important contributors to human SLE. Therapies blocking TLR7 itself and MyD88 may be more effective than therapies blocking germinal centers in SLE patients due to increased TLR7 signaling.

\section{Material And Methods}

\section{Mice}

Mice were bred and maintained in specific-pathogen-free conditions at the Australian National University (ANU), Canberra, Australia. Experimentation was performed according to the regulations approved by the local institution ethics committee, including the Australian National University's Animal and human Experimentation Ethics Committee. 


\section{Generation of the TIr7 and Rnaseh2b mutant mouse strains}

TIr7Y264H and deficient mice as well as Rnaseh2b-deficient knockout mice were generated using CRISPR-Cas9 mediated gene editing technology (34). Genomic sequences were obtained from Ensembl (Ensembl.org) and compared to ascertain conservation of the sequences between mouse and human genes. Single guide RNA (sgRNA) and single stranded oligonucleotides were purchased from IDT with the following sequences:

TIr7Y264H sgRNA 5'-TATGGGACATTATAACATCG-3' and

Rnaseh2b-5'-CTTTTAGTGCCACCACAGTT-3'

TIr7Y264H ssOligo: 5'GTCAATGAATTGAAAGCATTGTCATGGATCTGTAAGGGGGAATTATTTTCACACGGTGTACACGGATATGGGACATTATGACATCGAGGGCAATTTCCACTTAGGTCAAG $3^{\prime}$

3-4 week old C57BL/6Ncrl mice mated with $\mathrm{C} 57 \mathrm{BL} / 6 \mathrm{Ncrl}$ males. Pseudopregnant $\mathrm{CFW} / \mathrm{crl}$ mice were superovulated and mated with stud males. After detection of a vaginal plug, the fertilized zygotes were collected from the oviduct and Cas 9 protein ( $50 \mathrm{ng} / \mu \mathrm{l})$ was co injected with a mixture of sgRNA ( $2.5 \mathrm{ng} /$ $\mu \mathrm{l})$ and ssOligo $(50 \mathrm{ng} / \mu \mathrm{l})$ into the pronucleus of the fertilized zygotes. After the micro-injection of the eggs, the zygotes were incubated overnight at $37^{\circ} \mathrm{C}$ under $5 \% \mathrm{CO}_{2}$ and two-cell stage embryos were surgically transferred into the uterus of the pseudopregnant $\mathrm{CFW} / \mathrm{Crl}$ mice. The primers designed to amplify these regions are: TIr7_Y264H_F 5'- TGAAACACTCTACCTGGGTCA-3', _ TIr7_Y264H_R 5'- GCCTCCTCAATTTCTCTGGC-3' Rnaseh2b-F 5'GCAAGACCATCCCTACTCCA-3' and Rnaseh2b-_R 5'-AACACCTGCCCACATCTGTA-3'.

\section{Human samples DNA sequencing}

Written informed consent was obtained as part of the Centre for Personalised Immunology program. The study was approved by and complies with all relevant ethical regulations of the Australian National University and ACT Health Human Ethics Committees, the University Hospitals Institutional Review Board, or by Renji Hospital Ethics Committee of Shanghai Jiaotong University School of Medicine. Saliva was collected in Oragene ${ }^{\mathrm{Tm}}$ DNA self-collection kits and purified using PrepIT ${ }^{T M}$ DNA purification kits (Oragene) and treated with Ribonuclease A (Qiagen). DNA samples were enriched with Human SureSelect XT2 All Exon V4 Kit and sequenced by Illumina HiSeq 2000 (Illumina, Inc.). Bioinformatic analysis was performed at JCSMR, ANU as previously described (34).

\section{Human PBMC preparation}

PBMCs were isolated using Ficoll-Paque (GE Healthcare Life Sciences) gradient centrifugation and frozen in Fetal Bovine Serum (FBS, Gibco) with 10\% DMSO (Sigma Aldrich).

\section{Flow cytometry}

Single cells suspensions were prepared from mouse spleens or thawed PBMCs, and individual subsets analysed by flow cytometry. The primary antibodies used for mouse tissues included: SiglecH-APC (\#551, Biolegend), IgD-FITC (\#405718, Biolegend), IgD-PerCP Cy5.5 (\#11-26c.2a, BD Pharmingen), CD3-A700 (\#17A2, BioLegend), CD19- BUV395 (\#1D3, BD Horizon), CD138-PE (\#281-2, BD Pharmingen), PD1-BV421 (\#29F.1A12, BioLegend), CCR7-PerCP Cy5.5 (\#4B12, BioLegend), CD8-BUV805 (\#53-6.7, BD Horizon), CD19-BV510 (\#6D5, BioLegend), CD4-BUV395 (\#6K1.5, BD Horizon) CD21/35-BV605 (\#7G6, BD Horizon), CD45.1-BV605 (\#A20, BioLegend), CD45.1-BV711 (\#A20, BioLegend), CD45.1-PB (\#A20, BioLegend, TLR7-PE (\#A94B10, BD Pharmingen), CD23-BV421 (\#B3B4, BioLegend), CXCR3-PE (\#CXCR3-173, BioLegend), CD19-A700 (\#eBio1D3, Invitrogen), FoxP3-FITC (\#FJK-16s, Invitrogen (eBioscience), FoxP3-PECy7 (\#FJK-16s, Invitrogen eBioscience), IgM-FITC (\#II/41, BD Pharmingen), IgM-PECy7 (\#II/41, Invitrogen), CD44-FITC (\#IM7, BD Pharmingen), CD44-PB (\#IM7, BioLegend), CD95 (FAS)-BV510 (\#Jo2, BD Horizon), BCL6-A467 (\#K112-91, BD Pharmingen), CD11b-PerCP Cy5.5 (\#M1/70, BioLegend), IA/IE-BV421 (\#M5/114.15.2, BioLegend), CD11c-A647 (\#N418, BioLegend), CD11c-BV510 (\#N418, BioLegend), CD11c-FITC (\#N418, BioLegend), CD25-PE (\#PC62, BioLegend), B220-A647 (\#RA3-6B2, BD Pharmingen), B220-BUV395 (\#RA3-6B2, BD Horizon), B220-BUV737 (\#RA3-6B2, BD Horizon), CD98-PECy7 (\#RI.388, BioLegend), CD4-PECy7 (\#RM4-5, BD Pharmingen), CD25-A647 (\#PC61, Biolegend), CD4-A647 (\#RM4-5, Biolegend), CD11c-APC (\#HL3, BD Pharmingen), CD138-Biotin (\#281-2, BD Bioscience), CXCR5-Biotin (\#2G8, BD Bioscience), Streptavidin-BUV805 (BD Horizon), Streptavidin-BV510 (BioLegend), CD19-BV605 (\#6D5, Biolegend), B220-PE (\#RA3-6B2, Biolegend), BST2-PE (\#927, Biolegend), CD19-PE (\#6D5, Biolegend), IgD-PE (\#11-26c.2a, Biolegend), CD11b-PECy7 (\#M1/70, eBiosciences), Streptavidin-PECy (eBiosciences), CD4-PerCPCy5.5 (\#RM4-5, Biolegend), CD45.2-PerCPCy5.5 (\#104, BD Bioscience), CD3-Pacific Blue (\#HIT2, BD pharmingen). For human PBMCs: CD19-BV650 (\#HIB19, Biolegend), HLA-DR-BV510 (\#L243, Biolegend), CD 24-BV605 (\#ML5, Biolegend), CD56PECy7 (\#NCAM16.2, BD pharmingen), CD14-PerCP (\#MФP9, BD pharmingen), IgD-BV510 (\#IA6-2, Biolegend), CD123-PE (\#7G3, BD pharmingen), CD21-APC (\#B-ly4, BD pharmingen), CD11C-APC (\#B-ly6, BD pharmingen), CD16-APC-H7 (\#3G8, BD pharmingen), IgG-PECy7 (\#G18-145, BD pharmingen), CD10-PECF594 (\#HI10a, BD pharmingen), IgA-PE (\#IS11-8E10, Miltenyi Biotech), CD27-APC-EF-780 (\#O323, eBiosciences), IgM-EF450 (\#SA-DA4, eBiosciences), CD38PerCP-Cy5.5 (\#A60792, Beckman Coulter), CD93-PECy7 (\#AA4.1, Biolegend). Zombie aqua dye (BioLegend) or live dead fixable green (Thermos Fisher Scientific) was used for detecting dead cells. Cell Fc receptors were blocked using purified Rat anti-mouse CD16/CD32 (Mouse BD Fc Block ${ }^{\text {TM }}$ BD Biosciences) and then stained for $30 \mathrm{~min}$ at $4^{\circ} \mathrm{C}$, in the dark, with primary and secondary antibodies. Intracellular stains used the FOXP3 Transcription Factor Staining Buffer Set (eBioscience) as per the manufacturer's instructions. Samples were acquired on a Fortessa or Fortessa X-20 cytometer with FACSDiva (BD, Biosciences) and analyzed using FlowJo software v10 (FlowJo LLC). All FACS and microscopy work was carried out at the Microscopy and Cytometry Facility, Australian National University.

\section{Sanger sequencing}


Primers for human TLR7DNA sequencing were used at $10 \mu \mathrm{M}$ (primer sequences available on request). PCR amplification was carried out using Phusion Hot Start II DNA Polymerase II (Thermo Fisher Scientific) and under conditions recommended by the manufacturer. PCR amplicons were electrophoresed and excised bands purified using the QIAquick Gel Extraction Kit (Qiagen). Sanger sequencing was completed using Big Dye Terminator Cycle sequencing kit v3.1 (Applied Biosystems, Carlsbad) using the same primers used for PCR amplication. Sequencing reactions were run on 3730 DNA Analyze (Applied Biosystems) at the ACRF Biomolecular Resource Facility, Australian National University.

\section{Immunohistochemistry}

Liver, pancreas and kidneys were fixed in 10\% Neutral buffer formalin (NBF) solution, embedded in paraffin and stained by hematoxylin and eosin (H\&E).

\section{Bone Marrow Chimera experimentation}

For competitive bone marrow chimeras, Rag $1 \%$ mice were irradiated and injected intravenously with equal numbers of bone marrow cells from either WT or Kika CD45.2 and WT CD45.1 mice. Mice were given Bactrim in their drinking water for $48 \mathrm{hrs} \mathrm{before} \mathrm{injection} \mathrm{and} \mathrm{for} 6$ weeks following injection, and housed in sterile cages. Following 22 weeks of reconstitution mice were taken down for phenotyping by flow cytometry.

\section{B cell culture and Cell Trace Violet staining}

Single cell suspensions were prepared from kika, WT or TLR7 KO mouse spleens. B cells were magnetically purified using mouse B Cell Isolation Kit (Miltenyi Biotec), labeled with Cell Trace Violet (CTV, Thermo Fisher) and cultured for 72 hours in complete RPMI 1640 media (Sigma-Aldrich) supplemented with $2 \mathrm{mM}$ L-Glutamine (GIBCO), $100 \mathrm{U}$ penicillin-streptomycin (GIBCO), $0.1 \mathrm{mM}$ nonessential amino acids (GIBCO), $100 \mathrm{mM} \mathrm{HEPES} \mathrm{(GIBCO),} 55 \mathrm{mM} \beta$-mercaptoethanol (GIBCO) and $10 \% \mathrm{FBS}(\mathrm{GIBCO})$ at $37^{\circ} \mathrm{C}$ in $5 \% \mathrm{CO} 2$. For B cell receptor (BCR) stimulation, cells were cultured in $10 \mu \mathrm{g} / \mathrm{mL} \mathrm{AffiniPure} \mathrm{F}(\mathrm{ab}) \otimes$ fragment goat antimouse IgM, $\mu$ chain specific (Jackson Immuno Research) or $1 \mathrm{ug} / \mathrm{ml}$ each R837 (Invitrogen). CD93 expression was examined by sorting splenic B cells with CD19-PE (\#6D5, Biolegend), CD3-APCCy7 (\#17A2, Biolegend), CD93-APC (\#AA4.1, Invitrogen) and the viability stain 7-aminoactinomycin D (Molecular Probes, Invitrogen), cells were cultured with complete RPMI for 72 hours and stimulated with anti-mouse IgM or R837. Bone marrow was obtained from mice, the FC receptors blocked (Purified Rat Anti-Mouse CD16/CD32 (Mouse BD Fc Block ${ }^{\text {TM }}$ BD Biosciences) and cells stained and sorted with B220-PE (\#RA3-6B2, BioLegend), CD93-APC (\#AA4.1, Invitrogen) and the viability stain 7-aminoactinomycin D (Molecular Probes, Invitrogen). Cells were sorted on a FACS Aria II and cultured in Complete RPMI media.

\section{ADVIA blood analysis}

Orbital bleeds were performed on mice and blood samples were run on the ADVIA (Siemens Advia 1200).

\section{Western blotting}

Cytosolic extracts were prepared from 20-40 million splenocytes by lysis in TritonX100 buffer (0.5\% TritonX100, 20mM Tris. HCl pH7.4, 150mM NaCl, $1 \mathrm{mM}$ EDTA, $10 \%$ glycerol) and centrifuged. Cytosolic extracts were resolved on $8 \%$ SDS-polyacrylamide gels and probed with the relevant primary and secondary antibodies. Rabbit anti-TLR7 (D7; Cell Signaling Technology) and mouse anti mouseTLR7-PE (A94B10; BD Biosciences) were used at 1:1000, the actin monoclonal antibody (JLA20, Developmental Studies Hybridoma Bank, The University of lowa) was used at 1:5000. Membranes were developed with Clarity Western ECL Substrate (BioRad Laboratories).

\section{Dual luciferase assays}

RAW 264.7 cells were transfected with 245 ng of either pNIFTY (NF-KB luciferase; InvivoGen) or an IFN- $\beta$ luciferase reporter, pRL-CMV (100 ng; Promega) renilla luciferase control plasmid, $125 \mathrm{ng}$ of TLR7-HA plasmids (Genecopoeia Inc.) expressing the individual variants and MYD88. After overnight expression half the samples were stimulated with $1 \mathrm{ug} / \mathrm{ml}$ each R837 (Invitrogen) and R848 (Invitrogen) for 6 hrs and dual luciferase assays performed as previously described (34). For the MyD88 luciferase assays HEK293 cells were transfected with plasmids containing full length MYD88 (GenScript Biotech) or the MYD88 A6Pfs39* (GenScript Biotech) at 25, 50, 100 or $200 \mu \mathrm{g} / \mathrm{ml}$.

\section{Statistics}

Statistical analysis was carried out using R software version 3.6.1 (The R Foundation for Statistical Computing) and the Emmeans package. Mouse spleen mass data was analysed using two experiments as a blocking factor, followed by a pairwise estimated marginal means comparison of genotypes. Mouse cellular phenotyping, ELISAs, white blood cell and platelet count analysis were performed using a logged linear regression model, followed by a pairwise estimated marginal means comparison of genotypes. Purified B cell cultures were analysed using linear regression model followed by a pairwise estimated marginal means comparison of genotypes and stimulatory effect. Luciferase assays statistics were analysed using a one-way ANOVA with Tukey's multiple comparison (PRISM, GraphPad Software LLC). All data was graphed using PRISM.

\section{DNA, RNA, nRNP ELISAs}

Plates were coated with poly-L-lysine (Sigma Aldrich) before addition of $2.5 \mu \mathrm{g}$ of either DNA (D7290, Sigma), RNA (AM7120G, Thermo Fisher) or nRNP (SRC1000, Immunovision). Plates were then blocked in ELISA blocking buffer (PBS and 1\% BSA) for 2 hours at room temperature. Mouse serum was diluted 1:40 with ELISA coating buffer (0.05 M Sodium Carbonate anhydrous/Sodium Hydrogen Carbonate, $\mathrm{pH} 9.6$ ), and incubated in the ELISA plates overnight at $4{ }^{\circ} \mathrm{C}$. Plates were washed and goat anti-mouse IgG-AP antibody (Alkaline Phosphatase, Southern Biotech) added for 1 hour at $37^{\circ} \mathrm{C}$. Phosphatase substrate (Sigma 
S0942) was used as described by the manufacturer. Samples were read on an Infinite 200 PRO Tecan Microplate Reader (Tecan Group Ltd, Switzerland) at an absorbance of $405 \mathrm{~nm}$ and normalised to background absorbance at $605 \mathrm{~nm}$.

\section{HEp-2/Crithidia luciliae immunofluorescence}

Antinuclear antibodies (ANAs) and dsDNA were determined using Hep-2 and Crithidia luciliae slides (both from NOVA Lite), respectively. Serum was diluted 1:40 for HEp-2 slides and 1:20 for Crithidia slides and stained as described by the manufacturer using donkey anti-mouse IgG Alexa-488 (Moleclar Probes) as secondary antibody. Slides were imaged on an Olympus IX71 inverted bright-field/fluorescence microscope.

\section{Declarations}

Acknowledgments: We thank the personnel of the Australian Cancer Research Foundation Biomolecular Resource Facility (JCSMR) for Sanger sequencing; the Australian Phenomics Facility (APF) for animal care; Harpreet Vohra, Mick Devoy and Cathy Gillespie from the MCRF facility (JCSMR) for assistance with FACS sorting and imaging; Mark Koina for electron microscopy and Michael Gantier for critical reading of the manuscript. This work was funded by NHMRC fellowship and Program to CGV, NHMRC CRE to CGV and MCC, NHMRC Ideas grant to VA and an Elizabeth Greene Scholarship to GBr, The National Collaborative Research Infrastructure Strategy (NCRIS) via Phenomics Australia (GBu).

Author contributions: Conceptualization: G.J.B, V.A., C.G.V.; Methodology: G.B.; Formal analysis: G.J.B; Investigation: G.J.B. A.M., P.F.C., K.B., J.G.E., Y.Z., C.T., X.M., L.M., J.C., D.T., Y.H., J.B.; Resources: D.T., A.L., N.S., T.L., H.D., C.A.L., A.G., V.P., E.J.M, M.C.C., C.L.C., C.G.V; Data Curation: P.W., E.C., D.M.A., M.A.F.; Writing Original Draft: G.J.B, V.A., C.G.V.; Writing - Review \& Editing: V.A.; Visualization: G.J.B., P.F.C, E.C.; Supervision: V.A., C.G.V.; Project administration: G.J.B, V.A., C.G.V.; Funding acquisition V.A., G.G.V.

Competing interests: Authors declare no competing interests

Data and materials availability: Genomic data relating to the kindreds in Fig. 1 has been deposited in (to be provided); with accession number (to be provided)

\section{References}

1. J. K. Pritchard, Are rare variants responsible for susceptibility to complex diseases? American Journal of Human Genetics 69, 124-137 (2001).

2. T. A. Manolio et al., Finding the missing heritability of complex diseases. Nature 461, 747-753 (2009).

3. J. M. Alperin, L. Ortiz-Fernández, A. H. Sawalha, Monogenic Lupus: A Developing Paradigm of Disease. Frontiers in immunology 9, 2496-2496 (2018).

4. S. A. Jenks et al., Distinct Effector B Cells Induced by Unregulated Toll-like Receptor 7 Contribute to Pathogenic Responses in Systemic Lupus Erythematosus. Immunity 49, 725-739.e726 (2018).

5. P. T. Kuo et al., The Role of CXCR3 and Its Chemokine Ligands in Skin Disease and Cancer. Frontiers in Medicine 5, (2018).

6. B. P. dos Santos et al., TLR7/8/9 polymorphisms and their associations in systemic lupus erythematosus patients from Southern Brazil. Lupus 21, 302309 (2012).

7. N. Shen et al., Sex-specific association of X-linked toll-like receptor 7 (TLR7) with male Systemic Lupus Erythematosus. Proceedings of the National Academy of Sciences of the United States of America 107, 15838-15843 (2010).

8. C.-M. Wang et al., Genetic variations in toll-like receptors (TLRs 3/7/8) are associated with Systemic Lupus Erythematosus in a Taiwanese population. Scientific Reports 4, 3792 (2014).

9. P. Pisitkun et al., Autoreactive B cell responses to RNA-related antigens due to TLR7 gene duplication. Science 312, 1669-1672 (2006).

10. S. Subramanian et al., A TIr7 translocation accelerates systemic autoimmunity in murine lupus. Proceedings of the National Academy of Sciences of the United States of America 103, 9970-9975 (2006).

11. R. Berland et al., Toll-like receptor 7-dependent loss of B cell tolerance in pathogenic autoantibody knock-in mice. Immunity 25, 429-440 (2006).

12. A. Das et al., Follicular Dendritic Cell Activation by TLR Ligands Promotes Autoreactive B Cell Responses. Immunity 46, 106-119 (2017).

13. T. A. Schwickert et al., Ikaros prevents autoimmunity by controlling anergy and Toll-like receptor signaling in B cells. Nature immunology 20, $1517-1529$ (2019).

14. S. M. Al-Mayouf et al., Loss-of-function variant in DNASE1L3 causes a familial form of systemic lupus erythematosus. Nature Genetics 43 , $1186-1188$ (2011).

15. Z. Zhang et al., Structural Analyses of Toll-like Receptor 7 Reveal Detailed RNA Sequence Specificity and Recognition Mechanism of Agonistic Ligands. Cell Reports 25, 3371-3381.e3375 (2018).

16. S. J. Gibson et al., Plasmacytoid dendritic cells produce cytokines and mature in response to the TLR7 agonists, imiquimod and resiquimod. Cellular Immunology 218, 74-86 (2002).

17. S. S. Diebold, T. Kaisho, H. Hemmi, S. Akira, C. Reis e Sousa, Innate Antiviral Responses by Means of TLR7-Mediated Recognition of Single-Stranded RNA Science 303, 1529 (2004).

18. J. M. Lund et al., Recognition of single-stranded RNA viruses by Toll-like receptor 7. Proceedings of the National Academy of Sciences of the United States of America 101, 5598 (2004).

19. M. C. Woodruff et al., Extrafollicular B cell responses correlate with neutralizing antibodies and morbidity in COVID-19. Nature Immunology 21, 1506-1516 (2020). 
20. S. R. Christensen et al., Toll-like Receptor 7 and TLR9 Dictate Autoantibody Specificity and Have Opposing Inflammatory and Regulatory Roles in a Murine Model of Lupus. Immunity 25, 417-428 (2006).

21. C. M. Lau et al., RNA-associated autoantigens activate B cells by combined B cell antigen receptor/Toll-like receptor 7 engagement. The Journal of experimental medicine 202, 1171-1177 (2005).

22. K. M. Kelly-Scumpia et al., In vivo adjuvant activity of the RNA component of the Sm/RNP lupus autoantigen. Arthritis Rheum 56, 3379-3386 (2007).

23. J. K. Bell et al., Leucine-rich repeats and pathogen recognition in toll-like receptors. Trends in Immunology 24, 528-533 (2003).

24. M.-Y. Ahn et al., Toll-like receptor 7 agonist, Imiquimod, inhibits oral squamous carcinoma cells through apoptosis and necrosis. Journal of Oral Pathology \& Medicine 41, 540-546 (2012).

25. S. Margarete, P. S. Michael, The Antitumoral Mode of Action of Imiquimod and Other Imidazoquinolines. Current Medicinal Chemistry 14, 681-687 (2007).

26. Z. Zhang et al., Structural Analysis Reveals that Toll-like Receptor 7 Is a Dual Receptor for Guanosine and Single-Stranded RNA. Immunity 45, 737-748 (2016).

27. J. Wu et al., pDC Activation by TLR7/8 Ligand CL097 Compared to TLR7 Ligand IMQ or TLR9 Ligand CpG. Journal of Immunology Research 2019, 1749803 (2019).

28. C. Günther et al., Defective removal of ribonucleotides from DNA promotes systemic autoimmunity. The Journal of Clinical Investigation 125 , $413-424$ (2015).

29. K. J. Mackenzie et al., Ribonuclease H2 mutations induce a cGAS/STING-dependent innate immune response. The EMBO Journa/35, 831-844 (2016).

30. T. Li, Z. J. Chen, The cGAS-cGAMP-STING pathway connects DNA damage to inflammation, senescence, and cancer. The Journal of experimental medicine 215, 1287-1299 (2018).

31. Martin A. Reijns et al., Enzymatic removal of ribonucleotides from DNA is essential for mammalian genome integrity and development. Cell 149, 10081022 (2012).

32. N. V. Giltiay et al., Overexpression of TLR7 promotes cell-intrinsic expansion and autoantibody production by transitional T1 B cells. The Journal of experimental medicine 210, 2773-2789 (2013).

33. H. Wardemann et al., Predominant Autoantibody Production by Early Human B Cell Precursors. Science 301, 1374 (2003).

34. S. H. Jiang et al., Functional rare and low frequency variants in BLK and BANK1 contribute to human lupus. Nat Commun 10, $2201-2201$ (2019).

35. C. G. Vinuesa et al., A RING-type ubiquitin ligase family member required to repress follicular helper T cells and autoimmunity. Nature 435, 452-458 (2005).

\section{Figures}



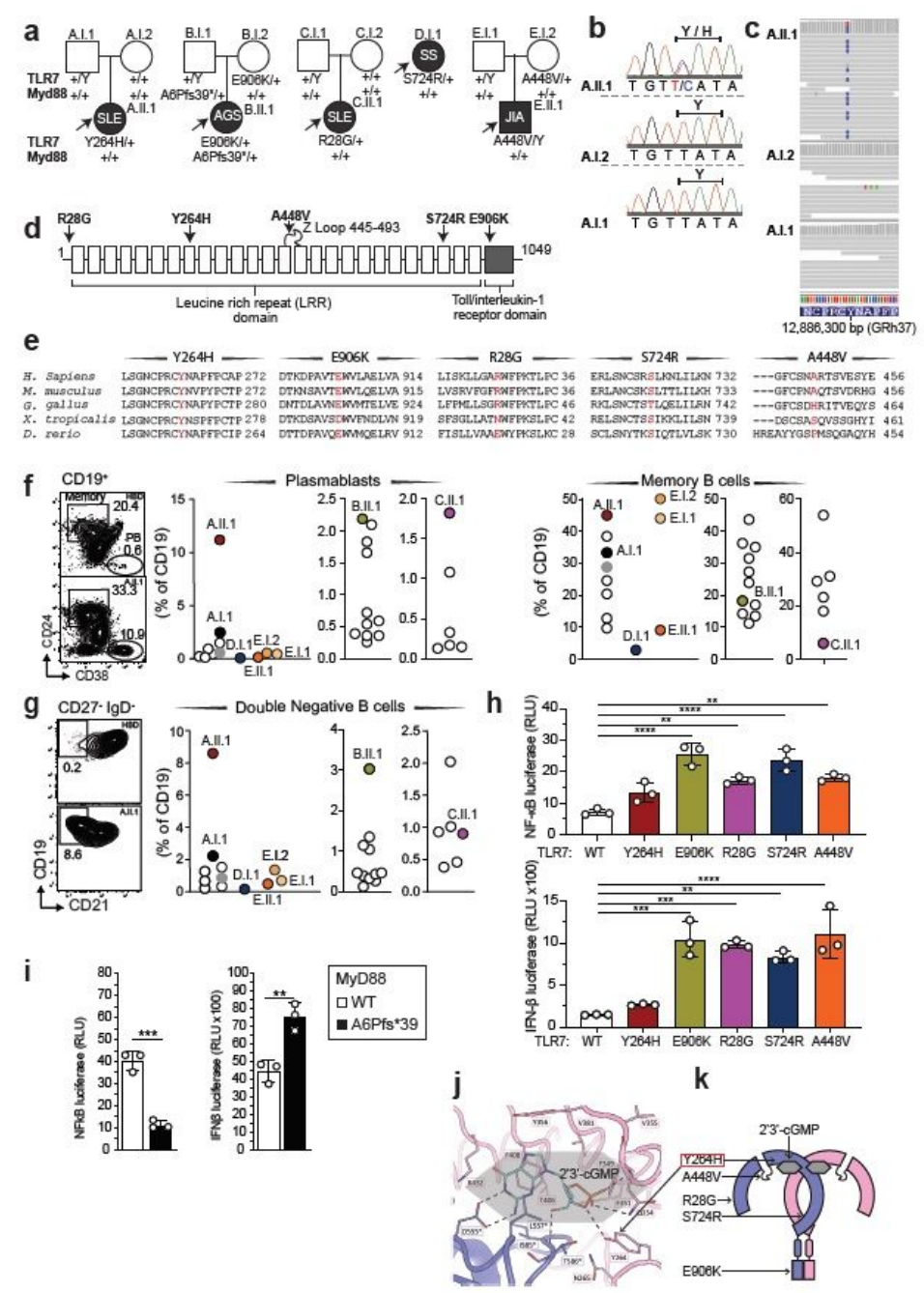

\section{Figure 1}

Identification of TLR7 variants in patients with systemic autoimmunity and PBMC phenotypic analysis. (a) Kindreds of probands (arrows) carrying TLR7 and/or MYD88 variants. (b-c) Sanger sequencing (b) and Integrative Genomics Viewer (IGV) image of the Y264H TLR7 de novo variant. (d) TLR7 leucine-rich repeat domains showing location of the TLR7 variants (23). (e) Phylogenetic conservation of TLR7 variants. (f-g) Flow cytometric plots showing (f) circulating plasmablasts (PB) (CD19+ CD38+ CD24-) and memory B cells (CD19+ CD38- CD24+) and (g) age associated B cells (ABC) (CD27- IgD- CD19hi CD21-). Grey dot without a border represents a healthy child control. White dots represent healthy adult controls. (h, i) NF-KB and IFN- $\beta$ luciferase assays (h) from expression of human TLR7 (WT, Y264H, E906K, R28G, S724R and A448V) in RAW 264.7 cells and (i) Myd88 (WT and A6Pfs 39 ) at 25, 50, 100 and 200 $\mu \mathrm{g} / \mathrm{mL}$ in HEK293 cells. (j) Model showing 2'3'-cGMP endogenous ligand bound to TLR7 and hydrogen bonding to Y264 at the small molecule binding site (modified from (15)). (k) Diagram of active dimerized TLR7 bound to 2'3'-cGMP. These results are representative of two experiments for patient PBMC phenotyping of Family $A$ and one for the remaining families. The luciferase assays bars show means and are representative of three NF-KB assays and three IFN- $\beta$ assays. ${ }^{\star} p$ $<0.05 ;{ }^{* \star} p<0.01, * * * p<0.001{ }^{* \star * *} p<0.0001$. 
a

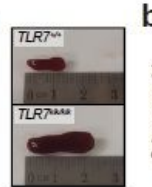

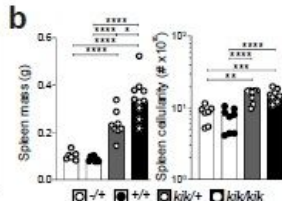

d

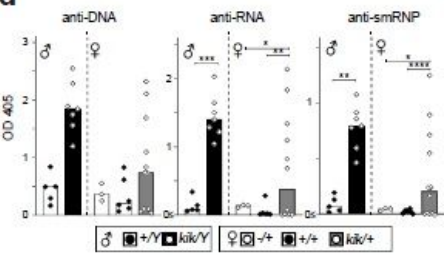

C

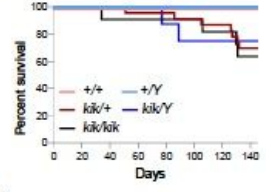

e

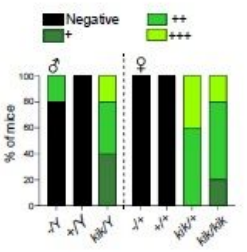

f

g

h
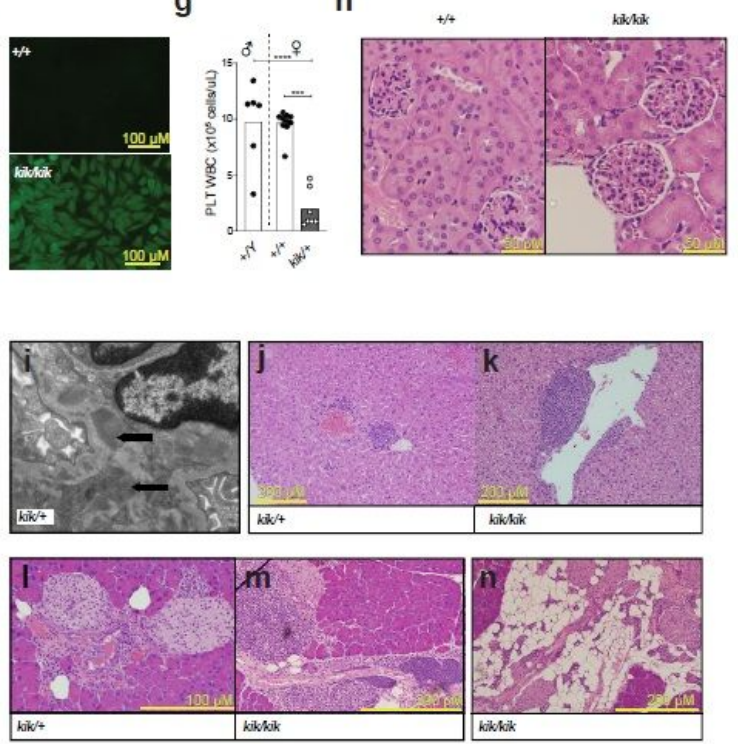

\section{Figure 2}

Kika mice have reduced survival, develop autoimmune symptoms and end organ damage. (a) Spleens from female wild type (TIr7+/+) and homozygous (Tlr7kik/kik) mice. (b) Median spleen mass and cellularity. (c) Survival of male and female kika mice. $T / r 7+/+n=12, T / r 7+/ k i k n=25, T l r 7 k i k / k i k n=11$, TIr7+/Y n = 11, TIr7kik/Y n = 7. (d) Antibodies to ssDNA (ANA), ssRNA and smRNP in serum from 12 wk-old male and female kika mice. (e, f) HEp-2 Immunofluorescence showing (e) quantification of ANAs in 12 wk-old kika mice and (f) pattern. (g) Platelet count in 18 wk-old female TIr7kik/+ mice. (h, i) H\&E staining (h) and electron microscopy (i) of kidneys from 6-month-old kika and control mice; black arrows show immunoglobulin deposits. (j-n) H\&E staining of liver (j, k) and pancreas (I-n) from 12 wk-old kika mice. Bars represent medians and each dot a single mouse. Results are representative of four experiments for spleen mass and cellularity, three for serum ELISAs, one for platelet analysis and three for organ H\&E analysis. ${ }^{*} \mathrm{p}<0.05$; ${ }^{\star \star} \mathrm{p}<0.01$, ${ }^{\star \star \star} \mathrm{p}<$ $0.001^{* * \star *} p<0.0001$. 
a

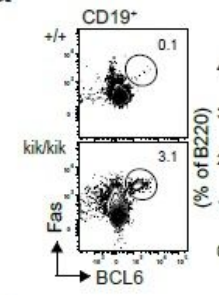

C
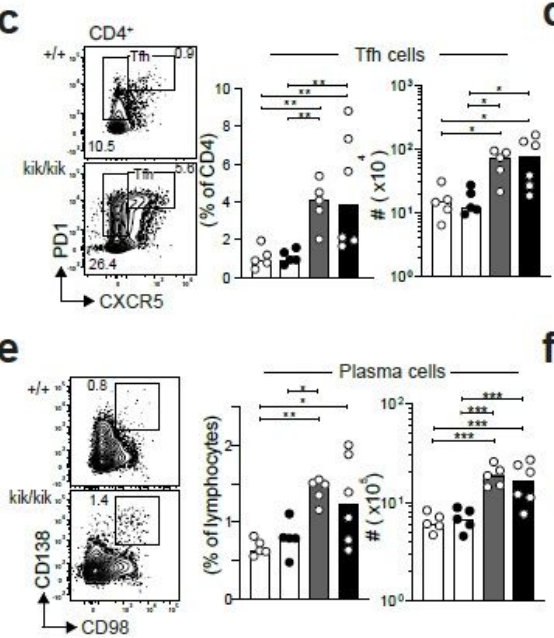

f

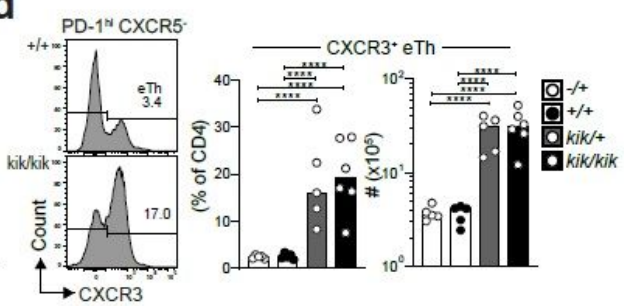

b
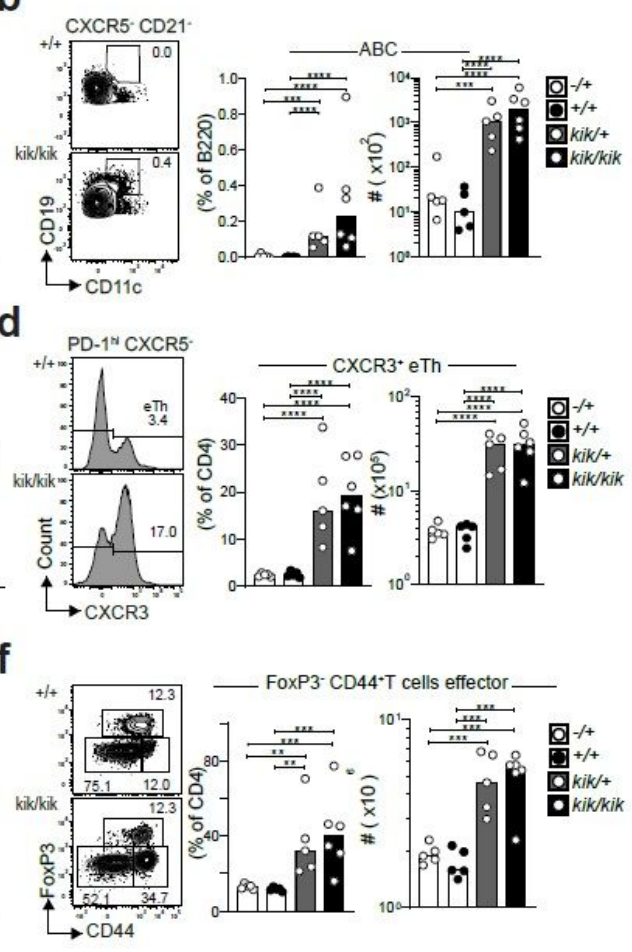

g

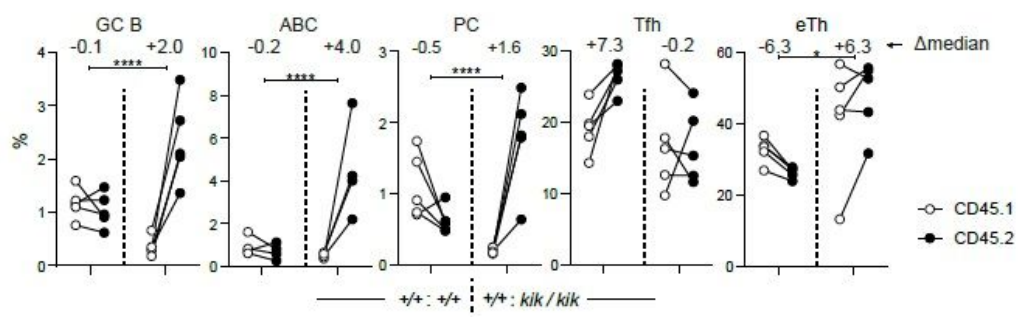

Figure 3

Cell-intrinsic expansion of ABCs and GC B cells in kika mice. Flow cytometric plots and quantification of splenic kika mice showing (a) GC B cells (CD19+ CD95+ BCL6+), (b) ABCs (B220+ CD21- CXCR5- CD19hi CD11c+), (c) T follicular helper cells (Tfh, CXCR5+ PD1hi), (d) extrafollicular (eTh, CD4+ CXCR5- PD1+ CXCR3+), (e) plasma cells (PC, CD138+ CD98+) and (f) CD4 effector T cells (CD4+ FoxP3- CD44+) at 12 weeks of age). (g) Quantification of the indicated splenic from mixed bone marrow chimeric mice containing a 1:1 ratio of control TIr7+/+ CD45.1/TIr7+/+ CD45.2 or TIr7+/+ CD45.1/TIr7kik/kik CD45.2 bone marrow. Bars represent medians and each dot a single mouse. These results are representative four splenic phenotyping and one experiment for chimera analysis. ${ }^{\star} p<0.05 ;{ }^{* \star} p<0.01,{ }^{\star \star \star} \mathrm{p}<0.001{ }^{\star \star \star \star} \mathrm{p}<0.0001$. 
a

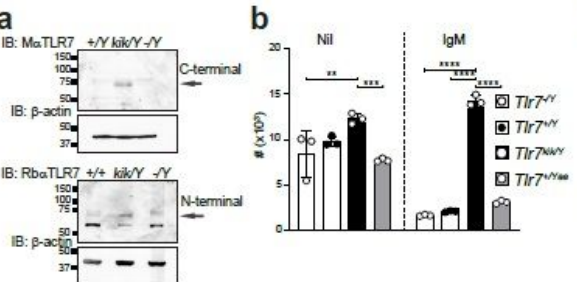

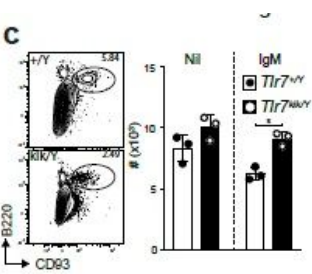
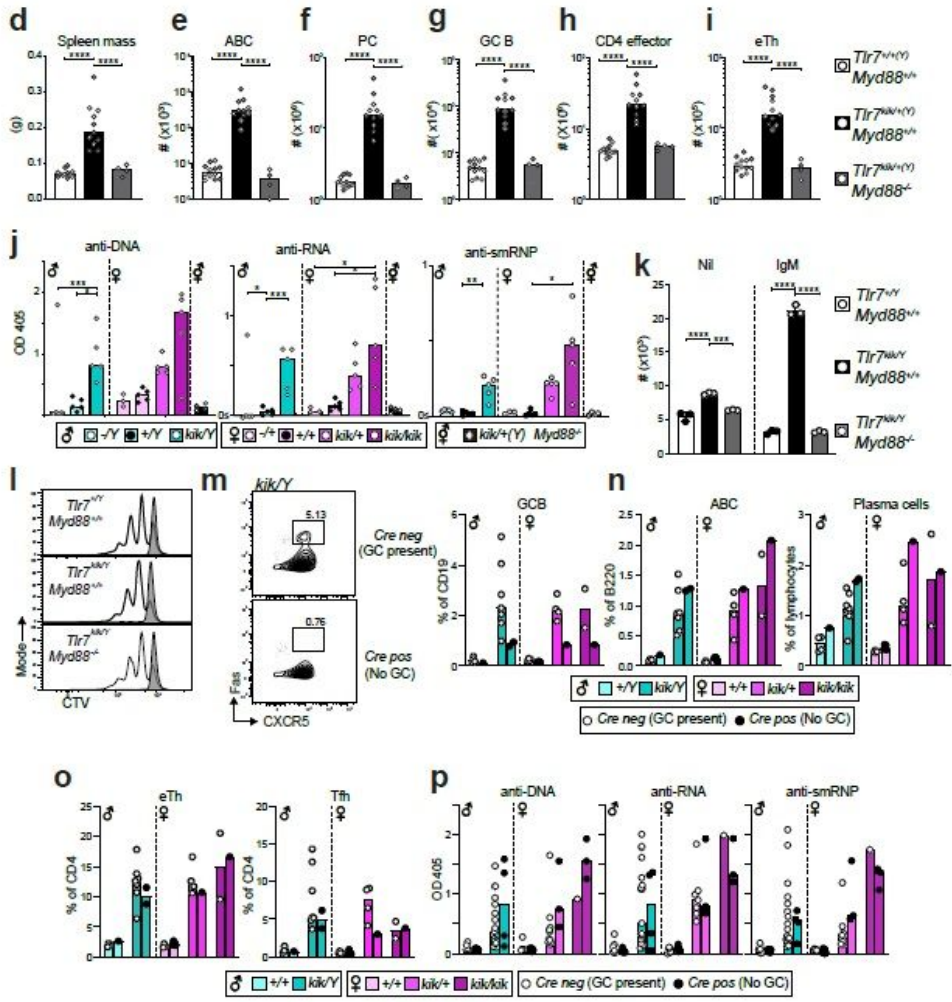

Figure 4

Constitutive activation of TLR7 promotes aberrant B cell survival and extrafollicular autoimmunity. (a) Western blots showing TLR7 expression in splenocytes from mice of the indicated genotypes. (b-c) Survival of (b) MACS-purified splenic B cells and (c) FACS sorted bone marrow immature B cells (B220int CD93+) cultured with or without u-IgM for 72 hours, from male mice of the indicated genotypes. (d) Spleen mass and (e-i) flow cytometric quantification of (e) $A B C s$ (B220+ CD21- CXCR5- CD19hi CD11c+), (f) plasma cells (PC, CD138+ CD98+), (g) GC B cells (GC B, CD19+ CD95+ BCL6+), (h) CD4 effector/memory T cells (CD4+ FoxP3- CD44+) and (i) extrafollicular helper cells (eTh, CD4+ CXCR5- PD1+ CXCR3+) from splenocytes of 12 wk-old male (grey) and female (white) kika mice either sufficient or deficient in Myd88. (j) Auto-antibodies to ssDNA (ANA), ssRNA and smRNP from 12 wk-old kika mice either deficient or sufficient in Myd88. (k) Survival and (I) proliferation of CTV-labeled splenic B cultured with u-lgM (white) compared to unstimulated cells (grey) for 72 hours. ( $\mathrm{m}-\mathrm{o}$ ) Flow cytometric plots and quantification of splenic (m) GC B cells (GCB, CD19+ CD95+ CXCR5+), (n) ABC, PC and (o) eTh and Tfh from 10 wk-old kika or control littermates either deficient (Bcl6flox/flox.CD23Cre) or sufficient (Bcl6flox/flox) in GCs. (p) Serum autoantibodies to ssDNA, ssRNA and smRNP in the same mice as in m-o. Bars represent medians and each dot a single mouse. Results are representative of three experiments for protein expression by Western blotting, B cell in vitro cultures, one BM B cell culture, one experiment for crosses to Myd88-/-, and two experiments for crosses to Bcl6flox/flox.CD23Cre. *p < $0.05 ; * \star p<0.01, * * * p<0.001 * \star \star * p<0.0001$

\section{Supplementary Files}

This is a list of supplementary files associated with this preprint. Click to download.

- SupplementaryInformation.pdf 